\title{
Control del arrastre de sedimentos post-incendio: efectividad de fajinas en un bosque seco montañoso (Salta, Argentina)
}

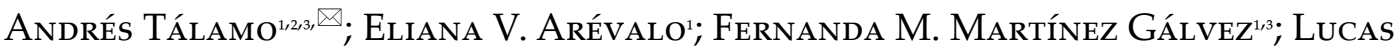 \\ A. Garibaldi ; Salomé C. Harmann ${ }^{*}$; Marina A. Díaz Leguizamón ${ }^{1}$ \& Joanna \\ CROCE ${ }^{2 / 3}$ \\ ${ }^{1}$ Facultad de Ciencias Naturales, Universidad Nacional de Salta. ${ }^{2}$ IBIGEO-Instituto de Bio y Geociencias del NOA. ${ }^{3}$ \\ CONICET, Consejo Nacional de Investigaciones Científicas y Técnicas. ${ }^{4}$ Instituto de Investigaciones en Recursos Naturales, \\ Agroecología y Desarrollo Rural (IRNAD), Sede Andina, Universidad Nacional de Río Negro (UNRN) y Consejo Nacional de \\ Investigaciones Científicas y Técnicas (CONICET). San Carlos de Bariloche, Río Negro, Argentina.
}

\begin{abstract}
Resumen. Los incendios forestales en bosques montañosos pueden modificar las tasas de infiltración y de escorrentía, lo cual aumenta el arrastre de sedimentos ladera abajo. Por lo tanto, es urgente diseñar e implementar técnicas de control de sedimentos en laderas incendiadas. Una metodología sencilla y económica es la construcción de fajinas (i.e., una empalizada de forma cilíndrica construida con ramas trenzadas). Nuestro objetivo fue evaluar la efectividad de colocar distintos números de fajinas $(0,2$ y 3$)$ en distintas posiciones de la ladera para controlar el arrastre de sedimentos en una ladera quemada (Serranía de Tres Cerritos, SaltaArgentina). Encontramos que a mayor número de fajinas, y ubicándolas en las partes bajas e intermedias de la ladera, el perfil de cenizas fue más profundo, lo que evidencia un mejor control del arrastre de sedimentos. Si bien el costo logístico de construir un sistema con hileras de 3 fajinas es un $50 \%$ más alto que otro con 2 hileras de fajinas, la ganancia en el control de sedimentos al trabajar con 3 fajinas fue superior en un $64 \%$. Recomendamos implementar un sistema con 3 (o más) fajinas para controlar el arrastre de sedimentos y la erosión del suelo en laderas quemadas en próximos eventos de incendios en bosques secos de montaña.
\end{abstract}

[Palabras clave: acumulación de cenizas, escorrentía, fajinadas, fuego, pérdida de suelo]

\begin{abstract}
Aвstract. Post-fire soil erosion control: using "fajinas" in a dry mountain forest (Salta, Argentina). Wildfires in mountain forests can trigger changes in rates of infiltration and runoff, increasing sediment flow downhill. Therefore, it is urgent to design and implement soil erosion control techniques on burned slopes. A simple and economic method is the construction of "fajinas" (cylindrical palisade built with braided branches). Our objective was to evaluate the effectiveness of the "fajinas" method in controlling sediment trawling on a burned slope located in the Serranía de Tres Cerritos (Salta, Argentina). We experimentally evaluated the effect of placing different numbers of "fajinas" (0,2 and 3) and in three different positions on a burned slope (high, medium and low) on the amount of ash retained (measuring ash profile height). We found that the greater the number of "fajinas", the deeper the ash profile was, showing a better control of sediment trawling. In addition, "fajinas" located in the lower or middle position of the slope controlled more effectively the sediment drag. Both factors acted independently. Although the cost/work of the construction of a control system with rows of 3 "fajinas" is greater (by 50\%) compared to a system with only 2 rows of "fajinas", the gain observed in the control of sediments exceeded $64 \%$ when working with 3 "fajinas". This justifies the recommendation of implementing systems with 3 "fajinas" as to control sediment trawling and soil erosion on slopes burned in future wildfire events in dry mountain forests.
\end{abstract}

[Palabras clave: ash accumulation, run-off, soil loss, wildfires]

\section{INTRODUCCIÓN}

El control de la erosión del suelo en bosques montañosos incendiados es una preocupación global de conservación y manejo. Los incendios forestales pueden modificar propiedades clave del suelo y alterar los procesos hidrológicos que allí ocurren. Por ejemplo, las temperaturas elevadas y la pérdida de la cobertura vegetal reducen la capacidad de infiltración (Shakesby and Doerr 2006; Moody et al. 2013; Moody and Ebel 2014) y aumentan la escorrentía y la erosión del suelo (Tálamo et al. 2016), con el consiguiente riesgo de arrastre de sedimentos a las viviendas ubicadas en el pie de monte de los sectores incendiados (Geertsema and Highland 2011; Parise and Cannon 2011). Por lo tanto, cobra importancia el diseño y la implementación de técnicas de control de erosión del suelo en las laderas recientemente quemadas a fin de minimizar las posibles consecuencias negativas de la alteración de los procesos hidrológicos. 
Para controlar la erosión hidrológica del suelo de una ladera incendiada, un primer paso es frenar o disminuir la velocidad de escurrimiento del agua de lluvia, y con ello su capacidad de arrastre de sedimentos (Huebl and Fiebiger 2005). Para esto existen técnicas que implican la construcción de estructuras que obstaculicen el escurrimiento, utilizando materiales como postes, piedras, mallas geotextiles, "mulching" y siembra de vegetación para acelerar la colonización del área quemada (Díaz Mendoza 2011; Prats et al. 2012, 2014, 2016). Una técnica económica consiste en la construcción de empalizadas trenzadas de ramas, de forma cilíndrica, llamadas fajinas (Medrano and de Sousa Borges 2007). Estas fajinas se colocan en el sentido de las curvas de nivel, con el objetivo de frenar o disminuir la velocidad del escurrimiento superficial, y constituyen un método sencillo y económico que se puede construir con material encontrado en el sitio.

La efectividad de un sistema de fajinas podría depender del número y de la ubicación de las líneas de fajinas colocadas en la ladera. Debido a que actúan como obstáculos mecánicos, es esperable que la capacidad de control de sedimentos aumente con el número de fajinas, ya que a mayor densidad de estructuras que obstaculicen el escurrimiento, mayor volumen de sedimentos retenidos. Sin embargo, también es probable que la efectividad tienda a un límite a partir del cual no se justifique seguir aumentando el número de líneas de fajinas. Por lo tanto, resulta interesante poner a prueba la efectividad del sistema de fajinas conformado por distintas densidades de fajinas. De manera similar, si las fajinas son colocadas en una zona elevada de la ladera, la efectividad en controlar la erosión podría no ser tan importante, ya que es poca la superficie que queda por ser erosionada aguas arriba de dicho punto. Por el contrario, las fajinas colocadas en una parte intermedia o baja de la ladera quemada pueden contener los sedimentos arrastrados de una mayor superficie de la ladera, lo que aumenta su efectividad. A pesar de que estos factores podrían ser clave en determinar el éxito de esta técnica de control del flujo de sedimentos en el suelo en bosques montañosos, no conocemos estudios que los hayan puesto a prueba por medio de experimentos.

En los últimos años, los bosques secos de montaña en Salta (noroeste argentino) fueron afectados por una sucesión de incendios forestales de orígenes diversos (Irigoin
2016). Se demostró experimentalmente que en estos bosques, la intensidad de la lluvia aumenta de manera significativa la pérdida de suelos en laderas quemadas (Tálamo et al. 2016). También se registraron eventos puntuales en los que, luego de un incendio forestal, las lluvias de verano provocaron un arrastre considerable de sedimentos que afectó a muchas viviendas ubicadas en el pie del cerro de las laderas quemadas (Barrio Portezuelo Norte, Salta Capital) (observación personal). Por lo tanto, resulta de gran interés poner a prueba metodologías sencillas para controlar o disminuir la erosión hídrica del suelo y disminuir así los riesgos de arrastre de sedimentos.

En este trabajo probamos experimentalmente la efectividad de las fajinas para controlar la erosión del suelo. En particular, evaluamos el efecto del número de fajinas (0, 2 y 3 fajinas) y de la posición altitudinal de las mismas (porción baja, media, y alta de la ladera) sobre la capacidad de controlar la erosión del suelo al frenar la bajada de sedimentos arrastrados por la lluvia natural en una ladera quemada (midiendo la altura del perfil de cenizas). Esperamos que el control del arrastre de sedimentos sea mayor al aumentar el número de fajinas, sobre todo en sectores bajos de la ladera.

\section{Materiales y Métodos}

\section{Área de estudio}

El sitio de estudio se encuentra sobre una serranía al este de la ciudad de Salta (Cerros de Tres Cerritos; $24^{\circ} 46^{\prime} 30^{\prime \prime} \mathrm{S}$ - 65²3'18" O), perteneciente a las Serranías de Mojotoro. La ladera estudiada se incendió en agosto de 2015, en plena época seca y en una semana de viento muy cálido y seco (viento Zonda). La región pertenece a la formación conocida como Bosques Secos Estacionales. El estrato arbóreo está dominado por un bosque de cebil colorado (Anadenanthera colubrina (Vell.) Brenan), acompañado por algunos árboles representantes de la de selva de transición, como Palo Borracho (Ceiba chodatii (Hassl.) Ravenna), Papaya de monte (Carica quercifolia (A. St.-Hil.) Hieron.), Horco quebracho (Schinopsis lorentzi Griseb) y Horco cebil (Parapiptadenia excelsa (Griseb.) Burkart)(Novara 1985). También se encuentran plantas herbáceas, enredaderas y helechos formando un denso sotobosque. El clima es templado. Las precipitaciones se concentran en el período estival, con un promedio de 700 
mm/año (Bianchi and Yánez 1992). Durante los tres meses del experimento hicimos una estimación de las precipitaciones por medio de un pluviómetro. En noviembre y diciembre de 2015 registramos $21.5 \mathrm{~mm}$ y $108 \mathrm{~mm}$, respectivamente, y en enero de 2016 medimos $126 \mathrm{~mm}$, lo cual arroja un promedio de $85.17 \mathrm{~mm} / \mathrm{mes}$. El registro histórico de precipitaciones de la ciudad de Salta para los mismos meses indica un promedio de 118.67 $\mathrm{mm} / \mathrm{mes}$ (Bianchi and Yánez 1992). El suelo es Hapludol fluvéntico, de textura franca, con bloques subangulares medios a finos y presencia de raíces (Nadir and Chafatinos 1990).

\section{Construcción de las fajinas}

Seleccionamos ramas de especies leñosas encontradas en el área de estudio. Sus diámetros oscilaron entre 4 y $15 \mathrm{~mm}$. Trenzamos las mismas en fajas de forma cilíndrica hasta construir una fajina de $15 \mathrm{~cm}$ de diámetro y $1 \mathrm{~m}$ de largo, aproximadamente. Construimos e instalamos las fajinas el 10 de noviembre de 2015 (Figura 1), antes de la época de lluvias; las sujetamos con hilo y las

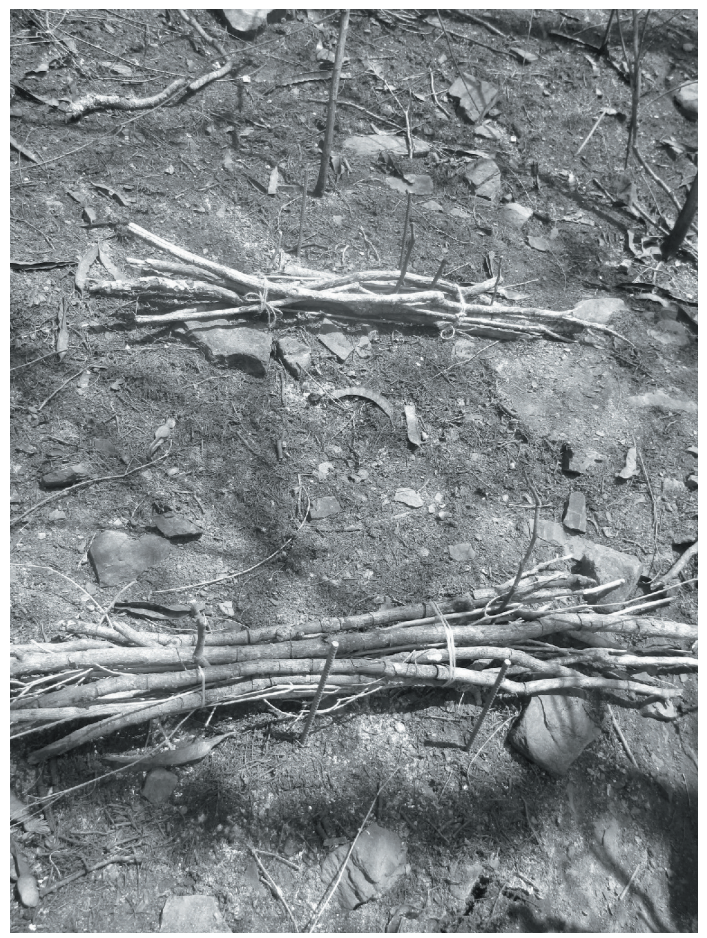

Figura 1. Imagen de una unidad experimental con 2 fajinas, instalada al principio del experimento en la parte alta de la ladera quemada de la Serranía Tres Cerritos, Salta Capital, Argentina.

Figure 1. Picture of one experimental unit, with 2 "fajinas" installed in the upper zone of the hillside in the Tres Cerritos Serranía, Salta, Argentina. fijamos al suelo con estacas de hierro de $6 \mathrm{~mm}$ de espesor y también con estacas de madera.

\section{Diseño experimental}

El experimento se realizó sobre la ladera incendiada, en sectores conla misma exposición (NO) y pendiente similar (media $=41.5 \%$; $\mathrm{CV}=10.6 \%$ ). Pusimos a prueba el efecto de dos factores: a) el número de fajinas (con 3 niveles: 0 fajinas [control], 2 fajinas [distanciadas a $1 \mathrm{~m}$ una de otra] y 3 fajinas [también distanciadas a $1 \mathrm{~m}])$, y b) la posición/altitud en la ladera (con 3 niveles: posición baja [a $10 \mathrm{~m}$ desde el comienzo de la ladera], posición alta [a $15 \mathrm{~m}$ antes de la cima de la ladera] y en una posición intermedia). No tuvimos en cuenta puntos inferiores a la posición baja ni superiores a la posición alta porque la pendiente se modificaba considerablemente. De este modo controlamos los posibles efectos del factor pendiente. La unidad experimental consistió en una superficie de suelo que recibió alguna de las combinaciones de los factores número de fajinas $x$ posición ( $3 \times 3 ; 9$ tratamientos). Los niveles "2 fajinas" y "3 fajinas" contaron con dos repeticiones en cada una de las tres posiciones de la ladera. Asociado a cada unidad experimental con 2 y 3 fajinas se seleccionó al azar (a la izquierda o a la derecha de cada conjunto de fajinas) la ubicación de la unidad experimental correspondiente al nivel "0 fajinas". Por lo tanto, disponemos de 24 unidades experimentales en total. Dado que realizamos el experimento en una sola ladera, las unidades experimentales ubicadas dentro de cada nivel de posición/altitud constituyen pseudo-repeticiones para dicho factor. Por tal razón, las conclusiones se acotarán a la ladera estudiada y discutimos la necesidad de aumentar el alcance del trabajo incorporando distintas laderas quemadas.

\section{Cuantificación del control de la erosión}

Medimos la acumulación de sedimentos al final de la época de lluvias (en abril de 2016). Para ello, en cada unidad experimental realizamos entre 2 y 6 cortes (submuestras) en el suelo, en la base de la fajina (a $10 \mathrm{~cm}$ ladera arriba de la fajina), y determinamos la altura de la capa de cenizas acumulada. Esta capa es claramente identificable de manera visual por el contraste de colores gris-marrón (Figura 2). Para realizar los análisis estadísticos, los valores de las submuestras se promediaron para cada unidad experimental. 


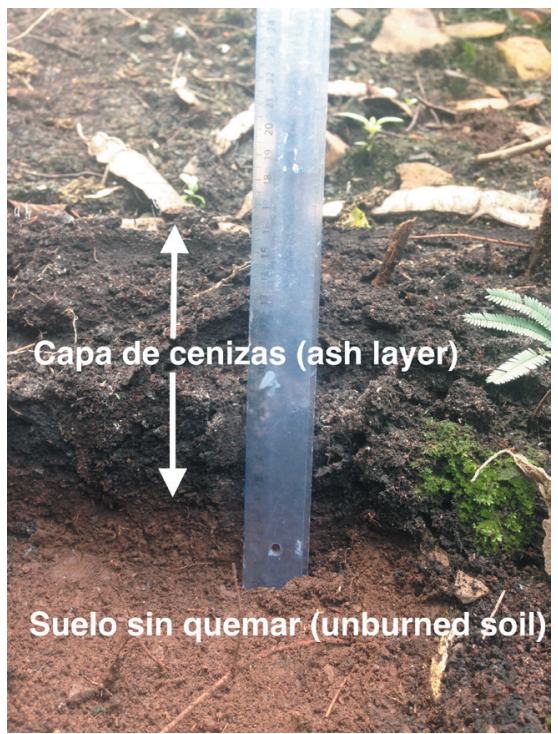

Figura 2. Medición de la profundidad de la capa de cenizas, que se distingue con claridad por su color oscuro. Serranía Tres Cerritos, Salta Capital, Argentina.

Figure 2. Measuring the depth of ash layer, easily distinguishable by its dark color. Serranía Tres Cerritos, Salta Capital, Argentina.

\section{Análisis de datos}

El efecto de ambos factores se evaluó mediante un análisis de varianza de dos factores con interacción. Los supuestos se verificaron gráficamente con diagramas de residuales. Todos los análisis se realizaron con el programa $\mathrm{R}$ ( $\mathrm{R}$ Development Core Team 2009) y se utilizó un nivel de significación prefijado de 0.05 .

\section{Resultados}

El número de fajinas y su posición en la ladera afectaron significativamente la profundidad del perfil de cenizas (fajinas: $\mathrm{F}_{2: 13}=29.00 ; P<0.001$; posición: $\mathrm{F}_{2: 13}=12.74$; $P=0.0009)$. Ambos factores actuaron de manera independiente (interacción: $\mathrm{F}_{4,13}=0.67$; $P=0.625$ ). La profundidad del perfil de cenizas acumulado en el tratamiento con 3 fajinas fue significativamente mayor que con 2 fajinas ( $24 \%$ de aumento), y ambos tratamientos tuvieron un perfil de mayor profundidad que los sitios sin fajinas ( $58 \%$ de aumento para 2 fajinas y $95 \%$ para 3 fajinas) (Figura 3). Por otro lado, la cantidad promedio de ceniza acumulada fue significativamente mayor en la posición baja ( $47 \%$ de aumento) e intermedia (62\% de aumento), en comparación con la posición alta (Figura 3). Independientemente de la zona considerada, la profundidad del perfil de cenizas aumentó de manera similar con el número de fajinas (Figura 3).

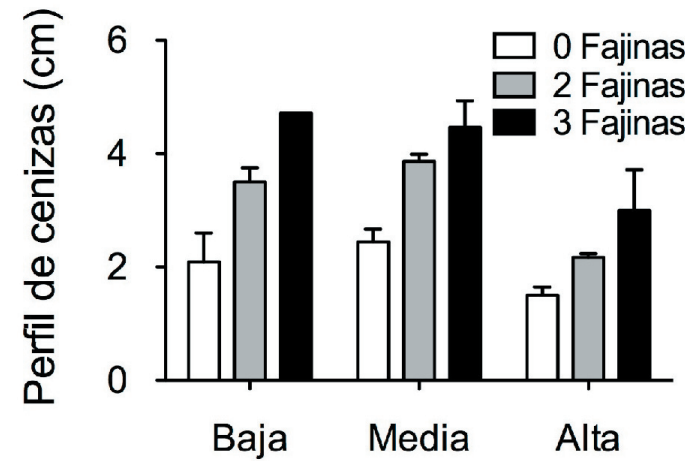

Figura 3. Profundidad media del perfil de cenizas (+1 EE) en 3 posiciones altitudinales (alta, media y baja) y en respuesta al número de fajinas, en una ladera quemada en la serranía Tres Cerritos, Salta Capital, Argentina.

Figure 3. Mean ash layer depth (+1 SE) in 3 altitudinal zones (baja: lower, media: intermediate and alta: upper) and in response of the number of "fajinas" treatment, in a burned slope in Tres Cerritos, Salta, Argentina.

\section{Discusión}

Nuestros resultados confirman la efectividad de las fajinas como técnica para disminuir el arrastre de sedimentos en la ladera incendiada. Más aún, nuestras predicciones se ven apoyadas por el hecho de que el control de la erosión fue mayor cuando colocamos un número mayor de fajinas, y en las partes bajas y medias de la ladera incendiada.

La acumulación de sedimentos lograda con estructuras que disminuyen o frenan la velocidad del agua de lluvia y, por lo tanto, del arrastre de sedimentos, fue documentado en varios ecosistemas mediterráneos (Wagenbrenner et al. 2006; Robichaud et al. 2008; Vásquez and Tapia 2011; Díaz-Raviña et al. 2012; Prats et al. 2012; 2012; Robichaud et al. 2013; Gómez-Rey et al. 2014; Prats et al. 2014). Sin embargo, en dichos estudios se comprobó la efectividad de técnicas diferentes a las fajinas (zanjas de infiltración, "hidromulching", "mulching" y siembra de vegetales, entre otros), y son pocos los estudios que usan estructuras similares. Una técnica similar a las fajinas consiste en la utilización de troncos o ramas gruesas de árboles muertos por el incendio, colocados en el sentido de las curvas de nivel. Esta técnica resultó efectiva en bosques incendiados de Estados Unidos, que contenían entre 7 y $32 \mathrm{~m}^{3} /$ ha de sedimentos (Wagenbrenner et al. 2006), especialmente en eventos breves de lluvia, pero no así con lluvias más frecuentes e intensas (Robichaud et al. 2008). Sin embargo, no conocemos estudios que hayan comparado la efectividad de trabajar con diferente densidad de estructuras que controlen el arrastre de sedimentos. 
Nuestro estudio demostró la conveniencia de trabajar con el máximo número de líneas de fajinas experimentado, evidenciado por la mayor acumulación de cenizas. Si se simplifica la heterogeneidad natural de los estudios de campo y se considera que la mayor cantidad de cenizas se acumuló $\sim 20 \mathrm{~cm}$ hacia arriba desde la fajina (observación personal), podemos extrapolar cuidadosamente nuestros resultados para estimar el volumen de cenizas acumulado ( $1 \mathrm{~m} \times 0.25 \mathrm{~m} \times$ profundidad promedio) y compararlo con el volumen equivalente sin fajinas (en otras palabras, estimar el efecto de cada tratamiento). El efecto de colocar 3 fajinas (diferencia promedio en altura del perfil de $1.92 \mathrm{~cm}$ ) equivaldría a un volumen promedio de $3.84 \mathrm{dm}^{3}$ de cenizas por metro lineal de fajinas, mientras que el efecto de colocar 2 fajinas (diferencia promedio de altura de $1.17 \mathrm{~cm}$ ) equivaldría a un volumen promedio de $2.34 \mathrm{dm}^{3}$ de cenizas por metro lineal. La diferencia entre los efectos de ambos tratamientos fue $1.50 \mathrm{dm}^{3}$ en un metro lineal de fajinas (un aumento de 64\%). Si bien desde el punto de vista logístico es más costoso construir un sistema con 3 fajinas que uno con 2 (un aumento del 50\% en el número de fajinas necesarias), consideramos que la ganancia en el volumen de cenizas retenido justifica incurrir en dicho costo. El incremento del perfil de cenizas a medida que aumentó el número de fajinas sugiere la conveniencia de usar un sistema de, como mínimo, 3 líneas de fajinas, además de seguir indagando sobre la conveniencia de trabajar con mayores números de fajinas.

Adicionalmente al número de fajinas, su ubicación en la ladera también constituye una decisión importante para garantizar la efectividad de la técnica y para maximizar el control de sedimentos, en casos de disponer de recursos limitantes. Como se planteó en nuestras predicciones, en la parte superior de la ladera se controló un menor flujo de sedimentos en comparación a las zonas medias y bajas de la ladera. A pesar de que nuestros resultados sugerirían la conveniencia de instalar el sistema de fajinas en las porciones medias y bajas de las laderas incendiadas, esta decisión podría no ser la más conveniente desde el punto de vista de la conservación del suelo. Esto es así por dos razones. Por un lado, las fajinas colocadas en la posición alta de la ladera, si bien retuvieron pocos sedimentos, podrían ser importantes para disminuir la escorrentía y la energía de arrastre del agua, lo que generaría una erosión menor ladera abajo. Por otro lado, al ubicar las fajinas sólo en la parte baja-media de la ladera se dejaría sin protección a una gran superficie de suelo ladera arriba. De este modo, en próximos eventos de lluvia, las fajinas colocadas en la parte baja-media lograrían frenar la bajada de sedimentos, pero no evitarían la erosión de la superficie de la ladera arriba de las fajinas. Por tal razón, se debería procurar cubrir con el sistema de fajinas diferentes posiciones altitudinales de la ladera incendiada.

El cambio climático global está modificando los regímenes de disturbios que ocurren en los bosques, en particular la frecuencia, la intensidad, la duración y el momento de los incendios forestales (Dale et al. 2001). Además, se predicen aumentos drásticos en el potencial de ocurrencia de incendios a nivel global (Liu et al. 2010). Por lo tanto, debemos estar preparados para recurrir a técnicas de control de erosión del suelo de laderas incendiadas con el fin de minimizar los impactos negativos del arrastre de sedimentos, sobre todo en sectores con viviendas ubicadas en el pie de monte. Experimentos de campo sencillos como el nuestro resultan necesarios para poner a prueba la efectividad de distintas alternativas de control de erosión de laderas incendiadas. Recomendamos fuertemente seguir profundizando en esta línea de investigación y abarcar un número mayor de laderas incendiadas para aumentar la generalidad de nuestras conclusiones.

Agradecimientos. Agradecemos a la Universidad Nacional de Salta por el subsidio № 2375 otorgado a través del Consejo de Investigación de la Universidad Nacional de Salta (CIUNSa) que nos facilitó la realización de los muestreos del presente trabajo.

\section{Referencias}

Dale, V. H., L. A. Joyce, S. Mcnulty, R. P. Neilson, M. P. Ayres, M. D. Flannigan, P. J. Hanson, L. C. Irland, A. E. Lugo, C. J. Peterson, D. Simberloff, F. J. Swanson, B. J. Stocks, and B. Michael Wotton. 2001. Climate Change and Forest Disturbances. BioScience 51:723-734.

Díaz Mendoza, C. 2011. Alternativas para el control de la erosión mediante el uso de coberturas convencionales, no convencionales y revegetalización. Ingeniería e Investigación 31:80-90.

Díaz-Raviña, M., A. Martín, A. Barreiro, A. Lombao, L. Iglesias, F. Díaz-Fierros, and T. Carballas. 2012. Mulching and seeding treatments for post-fire soil stabilisation in NW Spain: Short-term effects and effectiveness. Geoderma 191: 31-39. 
Geertsema, M., and L. M. Highland. 2011. Landslides: Human Health Effects. Pp. 380-395 in J. O. Nriagu (ed.). Encyclopedia of Environmental Health. Elsevier, Burlington.

Gómez-Rey, M. X., S. García-Marco, C. Fernández, A. Couto-Vázquez, and S. J. González-Prieto. 2014. Effects of post-fire soil stabilisation techniques on trace elements lost by erosion. International Journal of Wildland Fire 23:93-103.

Huebl, J., and G. Fiebiger. 2005. Debris-flow mitigation measures. Pp. 445-487 in Debris-flow Hazards and Related Phenomena. Springer Berlin Heidelberg.

Irigoin, N. 2016. Estadística de incendios forestales. Período 2014-15. Pp. 1-59 en Informe técnico, Subsecretaría de Desarrollo Foresto Industrial, Dirección de Producción Forestal, Buenos Aires, Argentina.

Liu, Y., J. Stanturf, and S. Goodrick. 2010. Trends in global wildfire potential in a changing climate. Forest Ecology and Management 259:685-697.

Medrano, V. C., and S. P. de Sousa Borges. 2007. Nuevos materiales para el control de la erosión tras los incendios forestales. Page 8. Regional Wildland Fire Networks, Seville, Spain.

Moody, J. A., and B. A. Ebel. 2014. Infiltration and runoff generation processes in fire-affected soils. Hydrological Processes 28:3432-3453.

Moody, J. A., R. A. Shakesby, P. R. Robichaud, S. H. Cannon, and D. A. Martin. 2013. Current research issues related to post-wildfire runoff and erosion processes. Earth-Science Reviews 122:10-37.

Nadir, A., and T. Chafatinos. 1990. Los suelos del N.O.A. (Salta y Jujuy). Universidad Nacional de Salta, Salta, Argentina.

Novara, L. J. 1985. Las unidades de vegetación del Noroeste del Valle de Lerma (Provincia de Salta, República Argentina). Documents Phytosociologiques 9:409-429.

Parise, M., and S. H. Cannon. 2011. Wildfire impacts on the processes that generate debris flows in burned watersheds. Natural Hazards 61:217-227.

Prats, S. A., L. H. MacDonald, M. Monteiro, A. J. D. Ferreira, C. O. A. Coelho, and J. J. Keizer. 2012. Effectiveness of forest residue mulching in reducing post-fire runoff and erosion in a pine and a eucalypt plantation in north-central Portugal. Geoderma 191:115-124.

Prats, S. A., M. C. Malvar, D. Catarina, S. Vieira, L. Macdonald, and J. J. Keizer. 2016. Effectiveness of hydromulching to reduce runoff and erosion in a recently burnt pine plantation in central Portugal. Land Degradation And Development 27:1319-1333

Prats, S. A., M. A. dos Santos Martins, M. C. Malvar, M. Ben-Hur, and J. J. Keizer. 2014. Polyacrylamide application versus forest residue mulching for reducing post-fire runoff and soil erosion. Science of the total environment 468: 464-474.

Robichaud, P. R., S. A. Lewis, J. W. Wagenbrenner, L. E. Ashmun, and R. E. Brown. 2013. Post-fire mulching for runoff and erosion mitigation. Catena 105:75-92.

Robichaud, P. R., J. W. Wagenbrenner, R. E. Brown, P. M. Wohlgemuth, and J. L. Beyers. 2008. Evaluating the effectiveness of contour-felled log erosion barriers as a post-fire runoff and erosion mitigation treatment in the western United States. International Journal of Wildland Fire 17:255-273.

Shakesby, R. A., and S. H. Doerr. 2006. Wildfire as a hydrological and geomorphological agent. Earth-Science Reviews 74:269-307.

Tálamo, A., R. Bermúdez, L. A. Garibaldi, and A. Chávez. 2016. Erosión y escorrentía en respuesta a lluvias simuladas en laderas quemadas y no quemadas (Salta-Argentina). Ciencia del suelo 34:105-111.

Vásquez, A., and M. Tapia. 2011. Cuantificación de la erosión hídrica superficial en las laderas semiáridas de la Sierra Peruana. Revista Ingeniería UC 18:42-50.

Wagenbrenner, J. W., L. H. Macdonald, and D. Rough. 2006. Effectiveness of three post-fire rehabilitation treatments in the Colorado Front Range. Hydrological Processes 20:2989-3006. 